\title{
17. THE SOLAR WIND - AN EXAMPLE OF A COSMICAL PLASMA \\ AND A STELLAR WIND
}

\author{
Introductory Report
}

(Sunday, September 14, 1969)

\author{
R. LƯST \\ Max-Planck-Institut für Physik und Astrophysik, Institut für extraterrestrische Physik, \\ Garching/München, Germany
}

\section{Introduction}

When Parker developed the theory of the solar wind, he suggested that other stars might also be surrounded by an expanding atmosphere. This suggestion has been worked out by Deutsch $(1958,1960,1961,1969)$ and by Weymann $(1960,1963)$, who discussed coronal evaporation as a possible mechanism for mass loss in red giants and used hydrodynamical equations for describing the phenomena. During the last few years detailed observations of the solar wind have been made, and the theoretical approach has also been further developed. Both might help us in our understanding of problems of a quasi-stationary mass loss of stars. But besides its relevance for the study of other stellar winds, the solar wind is important, as it is the best observed cosmical plasma available for guidance in the study of other cosmical, collisionless plasmas. What makes the interplanetary plasma so important is that beyond several solar radii from the Sun there are no collisions among the gas particles (one solar radius $\left[R_{\odot}\right]$ is $7.0 \times 10^{10} \mathrm{~cm} ; 1 \mathrm{AU}$ is $215 R_{\odot}$ ). Only near the Sun the collision rate is sufficiently high to maintain isotropy of the thermal particle motions and to produce ordinary fluid behavior. At larger distances from the Sun the fluid behavior is maintained by irregularities in weak magnetic fields and by micro-instabilities in the plasma.

It will be impossible to discuss here all aspects of the solar wind. I shall try to summarize those points which might be of particular interest. In Section 2 a summary of observational results will be given. In Section 3 theoretical models and in Section 4 transport of angular momentum will be discussed. We shall deal with waves and fluctuations in Section 5. Finally, in Section 6, the problem of the interaction of the solar wind with the surrounding interstellar medium will be studied.

For more details and also for the omitted subjects (such as interaction with cosmic rays, comets, planets, moon, and magnetosphere) I mention a number of review articles: Lüst (1962); Mustel' (1964); Parker (1963, 1965); Dessler (1967); Axford (1968); Ness (1968); Wilcox (1968); and in particular the two recent reviews by Hundhausen (1968b) and by Parker (1969) which helped greatly in preparing this Report.

\section{Observational Data}

A. QUIET STATE OF THE SOLAR WIND

Observational data about the quiet state of the solar wind were obtained mainly by 
Mariner 2 during its 1962 flight; by Vela 2, Vela 3, and Vela 4 in 1964 to 1967; and by IMP I in 1963 to 1964. The data were obtained near the orbit of the Earth (1 AU). According to the measurements by the Vela satellites, the flow or bulk velocity is near $320 \mathrm{~km} \mathrm{sec}^{-1}$ with a general inclination of $1.5^{\circ}$ from the radial direction in the sense of a co-rotation with the Sun. However, results from IMP I indicate an average inclination of $-15^{\circ}$ (anti-corotation) (Egidi et al., 1969). The proton density is about $5 \mathrm{~cm}^{-3}$; $\alpha$-particles amount to about $4.5 \%$ compared to protons (new observations on Explorer 34 by Ogilvie and Wilkenson, 1969, give about 5\% $\alpha$-particles) and other positive ions number less than $0.5 \%$ of the protons. The total positive-ion flux in the quiet solar wind is thus near $1.75 \times 10^{8}$ electronic charges $\mathrm{cm}^{-2} \mathrm{sec}^{-1} . \mathrm{He}^{+}$has been measured on certain occasions with an abundance of $n_{\mathrm{He}} / n_{\mathrm{p}} \leqslant 10^{-3} \cdot \mathrm{O}^{+6}, \mathrm{O}^{+5}$, and $\mathrm{O}^{+7}$ ions have been detected with variable relative abundances.

The solar-wind protons have a most probable temperature of about $4 \times 10^{4} \mathrm{~K}$ under quiet conditions. But even then the temperature varies considerably, occasionally dropping below $10^{4} \mathrm{~K}$. The $\mathrm{He}^{++}$ions have temperatures about four times higher than the protons. The electron temperature is near $10^{5} \mathrm{~K}$, which is about three times the proton temperature. The ion distribution functions are normally anisotropic, with the proton temperature along the interplanetary magnetic field lines about twice as high as normal to the lines. The electron distribution functions are less anisotropic than those of the protons. Both the electrons and protons are conducting heat outward from the Sun along the magnetic field lines, at rates of about $10^{-2}$ and $10^{-5} \mathrm{erg}$ $\mathrm{cm}^{-2} \mathrm{sec}^{-1}$, respectively.

The average magnetic field strength is about $5 \times 10^{-5} \mathrm{G}$. The magnetic energy density is much smaller than the kinetic energy density of the solar wind, and the magnetic field is carried along by the solar wind. The field either points away from the Sun or towards it in the form of an Archimedian spiral. The field lines have their origin in the Sun.

\section{B. TIME AND SPATIAL VARIATIONS OF THE SOLAR WIND}

The wind is highly variable in its velocity (which can go up to about $850 \mathrm{~km} \mathrm{sec}^{-1}$ ), in its direction $\left( \pm 15^{\circ}\right.$ ), in its temperature (up to $9 \times 10^{5} \mathrm{~K}$ ), and in its composition (up to $20 \% \alpha$-particles). Variations over the solar cycle have been observed. Also there often exist streams of hot, high-velocity plasma which recur at 27-day intervals. The magnetic field frequently shows a sector structure, which is correlated with the persistent high-velocity plasma streams. In addition a filamentary structure in the plasma and the magnetic field has been observed.

Short-time fluctuations - mainly observed by magnetometers since plasma detectors require larger sampling times - indicate plasma waves of various types, shock waves, and other discontinuities. They will be discussed in Section 5.

\section{Theoretical Description of the Large-Scale Properties of the Solar Wind}

The basic theory of the hydrodynamic expansion of extended stellar atmospheres has 
been developed by Parker (1963). The observations have confirmed his general ideas and are helping us now to improve the details of the different possible models. In his first models Parker demonstrated that from the Bernoulli equation alone - assuming an isothermal corona - a supersonic expansion at large distances would be implied if a small expansion velocity exists near the base of the corona and if the pressure at large distances from the Sun is small.

Assuming stationary conditions and spherical symmetry, we obtain from the equation of continuity

$$
r^{2} \varrho V=\text { const }
$$

( $r=$ distance from the origin, $\varrho=$ density, $V=$ radial velocity), and from the momentum balance, neglecting viscosity and magnetic field

$$
\varrho V \frac{\mathrm{d} V}{\mathrm{~d} r}=-\frac{\mathrm{d} p}{\mathrm{~d} r}-\varrho \frac{M_{\odot} G}{r^{2}}
$$

( $p=$ pressure, $M_{\odot}=$ solar mass, $G=$ gravitational constant) the relation

$$
\left[2-\frac{M_{\odot} G}{c_{s}^{2} r}\right] \frac{\mathrm{d} r}{r}=\left[\frac{V^{2}}{c_{s}^{2}}-1\right] \frac{\mathrm{d} V}{V} .
$$

Here $c_{s}=$ sound velocity; assuming adiabatic processes $\mathrm{d} p / \mathrm{d} \varrho=c_{s}^{2}$. Now $|\mathrm{d} p / \mathrm{d} r|>$ $\varrho M_{\odot} G / r^{2}$, because the corona is very hot and there exists no pressure far away from the Sun. Therefore, it follows from Equation (2) that $\mathrm{d} V / \mathrm{d} r>0$, i.e., the corona is expanding at an ever increasing speed. Equation (3) demonstrates that the expansion velocity will increase outward from subsonic to supersonic if $M_{\odot} G /\left(c_{s}^{2} r\right)>2$ for $V<c_{s}$ and if $M_{\odot} G /\left(c_{s}^{2} r\right)<2$ for $V>c_{s}$. Sonic velocity is reached at a critical point at $r=r_{c}$, where

$$
r_{c}=M_{\odot} G / 2 c_{s}^{2}
$$

For an isothermal, solar corona with $T=1.0 \times 10^{6} \mathrm{~K}$ and $c_{s}=1.7 \times 10^{7} \mathrm{~cm} \mathrm{sec}^{-1}$ we obtain from Equation (4) $r_{c}=3.5 R_{\odot}$. If the corona is not isothermal it will expand finally with supersonic velocity provided that the radial temperature dependence keeps the critical point at a finite distance. If we assume a polytropic relation $p \propto \varrho^{\alpha}$ this condition is satisfied as long as $\alpha$ remains smaller than $3 / 2$ (for an isothermal atmosphere $\alpha=1$ ). The solar gravitational force chokes or constricts the flow as does the converging section of a Laval nozzle (Clauser, 1960), and in this way it permits the development of sonic and supersonic flow. Also, the coronal temperature has to be within certain limits. It must be high enough so that the gravitational field cannot hold the corona in static equilibrium (i.e., $|\mathrm{d} p / \mathrm{d} r|$ has to be sufficiently large), but if the temperature were too high, the corona would be expanding only subsonically, since the influence of the gravitational field would be rather small [in Equation (3), $c_{s}$ would be very large]. The exact lower and upper bounds for the average temperature depend on radial variation in the temperature, which are determined by the thermal processes involved. The maximum temperature $T_{m}$ at which supersonic expansion can occur is 
determined by Equation (4) with $r_{c}=R_{\odot}$ and $c_{s}^{2}=2 \gamma k T / m$ ( $\gamma=$ ratio of specific heats, $k=$ Boltzmann constant, $m=$ mass of proton). However, since the temperature decreases towards the chromosphere, a lower temperature in the chromosphere may yield $r_{c}>1 R_{\odot}$ and the coronal expansion will always be supersonic.

To obtain the values of the density, the velocity, and the temperature near the orbit of the Earth, it is necessary to take into account also the energy equation, in which thermal conduction plays a significant role. However, a number of calculations with a two-fluid model (Sturrock and Hartle, 1966; Hartle and Sturrock, 1968) have shown that thermal conduction alone is not sufficient for supplying the necessary energy. One obtains a too-high electron temperature and a too-low ion temperature. Furthermore, as in one-fluid hydrodynamic models, the density of the wind at $1 \mathrm{AU}$ tends to be higher and the velocity lower than observed. As a consequence, besides conduction and convection, other forms of energy transport must be operative. During quiet conditions these other forms should be effective out to at least $r=3 R_{\odot}$, but during active periods (indicated by higher temperatures, high densities, and high wind velocities) the energy input should be enhanced out to the orbit of the Earth. The additional energy is presumably delivered by waves or by turbulence. The mechanism responsible for heating near the Sun might also be effective in the outer corona, since not all energy in the shock waves will be absorbed in the inner corona. In addition other waves might be heating, for in a collisionless plasma hydromagnetic waves behave different from those in a collision-dominated plasma. During quiet times the fast-mode of magnetoacoustic waves could heat effectively close to the Sun, as proposed by Barnes $(1968,1969)$. These waves would have frequencies small compared to local ion frequencies, and wavelengths long compared to local Larmor radii. Collisionless damping of hydromagnetic waves is probably important also in a number of other astrophysical phenomena, first of all for heating astrophysical plasmas. Although other kinds of plasma waves (e.g., ion cyclotron waves) are more efficient plasma heaters, in astrophysical situations most wave energy seems to exist in the hydromagnetic modes associated with large-scale plasma motions. Second, wave damping is intimately related to scattering of charged particles by turbulent plasma.

Barnes estimates that a flux of about $5 \times 10^{26} \mathrm{erg} \mathrm{sec}^{-1}$ propagates outwards into the outer corona in the form of fast-mode magnetoacoustic waves. Most of this energy is transformed into electron and ion thermal energy within a few $R_{\odot}$, and it is expected to increase the velocity of the wind at the orbit of the Earth. Beyond a few $R_{\odot}$, the surviving fast-wave energy (about $5 \times 10^{24} \mathrm{erg} \mathrm{sec}^{-1}$ ) is propagating parallel to the magnetic field, and hence it is not dissipated. This situation persists out to $r \approx 10 R_{\odot}$, where the solar field ceases to be radial. At this point the remaining waves will damp out, supplying additional energy which goes mainly to the protons because of the direction of wave propagation. Since the waves increase only the kinetic temperature parallel to the main magnetic field, the transverse temperature remains unchanged. In this way we can understand the observed anisotropies in the temperature. The higher electron temperature is most probably due to the larger electron heat conduction. 
The fast-mode magnetoacoustic waves are generated in the solar corona as remnants of processes occurring in the solar photosphere, such as the granulation or spicules. The waves will have damped out before reaching the orbit of the Earth. Therefore the waves observed at the orbit of the Earth - in particular those with shorter wavelengths (less than $10^{7} \mathrm{~km}$ ) - are generated in the solar wind and do not directly reflect processes occurring at the surface of the Sun. The wavelength spectrum at the orbit of the Earth may reflect some sort of equilibrium between generation and decay of hydromagnetic waves. The strong damping of waves provides a significant heat source for the solar wind.

However, waves generated by internal plasma instabilities cannot heat the wind, since they contribute only to the equalization of the anisotropies, and probably do not produce the long-wavelength waves of interest. Jokipii and Davis (1969) proposed that the interaction between streams, or sectors distributed in solar longitude, of different wind velocity would provide a dominant source for long-wavelength waves that heat the plasma. The velocity differences between such streams represent an energy source corresponding to a thermal velocity of a few hundred $\mathrm{km} \mathrm{sec}^{-1}$ which is certainly adequate to maintain the solar wind temperature around some $10^{5} \mathrm{~K}$. The energy source has also the required property of being variable. In addition it would heat the particles proportionally to their mass, since the interaction produces equal velocity spreads. Therefore we would expect that during perturbed times the ion temperatures are proportional to their mass. This would indeed explain the fact that during active times the temperature of $\alpha$-particles is about four times that of protons while during cold and quiet times the temperatures are more nearly equal. Another interesting feature of this model is that the sharpest fluctuations and strongest heating occurs within 2 to $3 \mathrm{AU}$ from the Sun, since the fluctuations should be generated as long as the stream-velocity differences persist and interact strongly. If there are, for instance, four fast streams distributed around the Sun with $\Delta V=100 \mathrm{~km} \mathrm{sec}^{-1}$ over the intervening $300 \mathrm{~km} \mathrm{sec}^{-1}$ slow streams, and each fast stream lags the preceding slow stream by about $45^{\circ}$, then the fast streams, if undeflected by the slow streams, would pass across the slow streams at about $3 \mathrm{AU}$. More gradual, very-long-wavelength fluctuations are confined to a region whose heliocentric radius has an upper limit of 15 to $30 \mathrm{AU}$. Beyond this point the wind should cool adiabatically, and both the magnetic structure and the velocity field seem likely to be quite uniform except perhaps for the short-wavelength fluctuations generated by the anisotropy of the temperature. This result is in agreement with recent observations of galactic cosmic rays which indicate that the boundary of sensible modulation by the solar wind is roughly at 5 to $10 \mathrm{AU}$ at solar minimum (Meyer et al., 1956; O'Gallagher and Simpson, 1967; Simpson and Wang, 1967). The propagation or diffusion of cosmic-ray particles in the solar system may be determined from the observed power spectrum of magnetic irregularities with wavelengths roughly equal to the particle cyclotron radius. These would be of the order of $10^{5}$ to $10^{7} \mathrm{~km}$ for cosmic-ray particles with energies between $10 \mathrm{MeV}$ and $10 \mathrm{GeV}$ per nucleon in the $5 \times 10^{-5} \mathrm{G}$ average interplanetary magnetic field. 
As just outlined the model calculations showed that thermal conduction and convection alone is not sufficient for producing the observed temperature, but that the damping of certain waves can explain the observed parameters in the solar wind. Direct evidence of the coronal gas before it enters the wind is contained in the observed state of ionization of oxygen. Calculations by Hundhausen et al. (1968), show that the state of ionization remains essentially fixed beyond about two solar radii since no more recombinations take place. In the solar wind $\mathrm{O}^{+6}$ is more abundant than $\mathrm{O}^{+7}$, implying that the temperature in the low corona is usually below about $2.2 \times 10^{6} \mathrm{~K}$. But sometimes the observations show $\mathrm{O}^{+7}$ several times more abundant than $\mathrm{O}^{+6}$, indicating the temperature of the coronal source region near $3 \times 10^{6} \mathrm{~K}$. The observations with the Vela space vehicle show, furthermore, that coronal sources at widely varying temperatures can give rise to a solar wind with the same low temperature $\left(\approx 10^{4} \mathrm{~K}\right)$ at $1 \mathrm{AU}$. This is another indication that the ion temperature in the interplanetary space is largely determined by processes affecting the particles well out in the interplanetary region.

Finally it should be mentioned that the observed $\mathrm{He}^{+}$is not consistent with the establishment of the ionization state of helium deep in the corona and thus must indicate some local interplanetary modifications of that state.

\section{The Transport of Angular Momentum}

\section{A. TRANSPORT OF ANGULAR MOMENTUM BY THE MAGNETIC FIELD}

So far only radial motions have been considered. Now we discuss also the azimuthal motions and their relation to the magnetic field. While the magnetic-energy density is small compared to the kinetic energy density at $1 \mathrm{AU}$, the situation is reversed near the Sun. There, assuming a $1 \mathrm{G}$ field, the magnetic energy density is larger than the thermal energy density even in quiet regions. Therefore the magnetic field will certainly influence the motions of the gas.

The large-scale picture of the magnetic field in the interplanetary space is determined by the outward motion of the solar wind, and the solar fields are extended out into the solar system. Due to the rotation of the Sun the field should have a spiral structure which is indeed observed. For the simple case of a constant radial wind velocity $V$ the field beyond $r_{0}$ is given by (Parker, 1958):

$$
\begin{aligned}
& B_{r}(r, \theta, \varphi)=B\left(\theta, \varphi^{*}\right)\left(\frac{r_{0}}{r}\right)^{2} \\
& B_{\varphi}(r, \theta, \varphi)=B\left(\theta, \varphi^{*}\right) \frac{r_{0}}{r} \frac{r_{0} \Omega}{V} \sin \theta \\
& B_{\theta}(r, \theta, \varphi)=0,
\end{aligned}
$$

when $B(\theta, \varphi)$ is the field at $r=r_{0}$, when $\theta$ and $\varphi$ represent the polar and azimuthal angles, when $\Omega\left(=2.5 \times 10^{-6}\right.$ radian $\left.\mathrm{sec}^{-1}\right)$ is the angular velocity of the Sun and when $\varphi^{*} \equiv \varphi+r \Omega / V$. Assuming a field of $1 \mathrm{G}$ at the surface of the Sun one obtains $3 \times 10^{-5} \mathrm{G}$ 
at the orbit of the Earth, giving an Alfvén speed $V_{A}=30 \mathrm{~km} \mathrm{sec}^{-1}$ for a particle density of 4 proton $\mathrm{cm}^{-3}$. Thus at $1 \mathrm{AU}$ the Alfvén speed is of the same order as the thermal velocity at a temperature of about $5 \times 10^{4} \mathrm{~K}$.

Near the Sun the magnetic field is sufficiently strong, and it will force the gas to co-rotate. In this way the gas will take away some angular momentum from the Sun. But the magnetic field, which has an azimuthal component $B_{\varphi}$, will also remove solar angular momentum. Before we discuss this problem in more detail, we shall estimate the torque exerted on the Sun by the Maxwell stress of the magnetic field (Parker, 1958). The total torque exerted on the Sun is given by

$$
Z=r^{3} \int_{0}^{\pi} \mathrm{d} \theta \sin ^{2} \theta \int_{0}^{2 \pi} \mathrm{d} \varphi \frac{B_{r} B_{\varphi}}{4 \pi} .
$$

If we assume that $B(\theta, \varphi)$ is uniform over the entire Sun with a field strength $B_{0}$, it follows that the total torque (and therefore the rate of outward transport of angular momentum) is given by

$$
Z=\frac{1}{3} r_{0}^{4} \frac{\Omega}{V} B_{0}^{2}=\frac{1}{3} \frac{\Omega}{V}\left(r^{2} B_{r}\right)^{2} .
$$

This rate is independent of $r$. Assuming $V=400 \mathrm{~km} \mathrm{sec}^{-1}$ and $B_{0}=2 \mathrm{G}$ (corresponding to $5 \times 10^{-5} \mathrm{G}$ at $1 \mathrm{AU}$ in the radial direction), the torque is $4 \times 10^{30} \mathrm{dyne} \mathrm{cm}$. If the Sun rotates rigidly its angular momentum is about $2 \times 10^{48} \mathrm{~g} \mathrm{~cm}^{2} \mathrm{sec}^{-1}$ and the outer convection zone might have about one tenth of this. Hence the characteristic deceleration time is $0.5 \times 10^{18} \mathrm{sec}$ or $2 \times 10^{10} \mathrm{yr}$ for the entire Sun and $2 \times 10^{9} \mathrm{yr}$ for the convection zone alone, as compared with the present age of $5 \times 10^{9} \mathrm{yr}$ of the Sun.

\section{B. TRANSPORT OF ANGULAR MOMENTUM BY THE OUTFLOWING GAS}

So far we have neglected any azimuthal motion and thereby any transport of angular momentum by the gas. While for the Sun this approximation is sufficient (as we will see), the situation is different for stars with higher rotational velocities and stronger magnetic fields. We will now discuss the study of this problem by Weber and Davis (1967a). Again stationary conditions, spherical symmetry and infinite electrical conductivity are assummed and the dynamical equations are considered only in the vicinity of the equatorial plane. The additional equations which have to be taken into account are, first,

$$
\operatorname{curl}[\mathbf{V} \times \mathbf{B}]=0
$$

and, second, the equation of motion in the $\varphi$-direction. The last one can be integrated yielding the sum of the angular momentum of the gas and the torque by the magnetic field. This sum, called $L$, must be a constant. Then,

$$
r V_{\varphi}-\frac{r B_{r} B_{\varphi}}{4 \pi \varrho V_{r}}=L=\Omega r_{A}^{2} .
$$


Here $V_{\varphi}=$ azimuthal velocity and $r_{A}$ the distance where $V_{r}=B_{r} / \sqrt{ }(4 \pi \rho)$. Thus $L$, the total angular momentum per unit mass, is determined by the position of the 'Alfven critical distance' $r_{A}$. The azimuthal velocity at this distance is of the order of the velocity of rigid rotation. At larger distances $V_{\varphi}$ has the form

$$
V_{\varphi} \approx \frac{\Omega r_{A}^{2}}{r}\left(1-\frac{V_{r A}}{V_{r \infty}}\right)
$$

where $V_{r A}$ and $V_{r \infty}$ are the radial velocities at $r=r_{A}$ and for large $r$ respectively. Weber and Davis used the following parameter values: $V_{r}=400 \mathrm{~km} \mathrm{sec}^{-1}, n=7$ proton $\mathrm{cm}^{-3}$, $B=5 \times 10^{-5} \mathrm{G}$ at $1 \mathrm{AU}$ and $\mathrm{B}=2.4 \mathrm{G}$ at $r=r_{\odot}$. Adopting a polytropic index $\alpha=1.229$, they find $V_{\varphi}=1 \mathrm{~km} \mathrm{sec}^{-1}$ at $1 \mathrm{AU}$. Three quarters of the angular momentum is carried away by the magnetic field and one quarter by the gas $\left(r_{A}=24.6 r_{\odot}\right.$ and $V_{\varphi}$ reaches a maximum with $V_{\varphi}=2 r_{\odot} \Omega$ at $r=11.5 r_{\odot}$ ).

\section{PROBLEMS FOR THE SOLAR CASE; APPLICATION TO OTHER STARS}

The Weber and Davis model predicts an azimuthal velocity of the solar wind near the Earth's orbit of about $1 \mathrm{~km} \mathrm{sec}^{-1}$ (conservation of angular momentum would yield only about $10^{-2} \mathrm{~km} \mathrm{sec}^{-1}$ ). The major part of the angular momentum is transported by the magnetic field. Therefore the simple picture outlined in Section $4 \mathrm{a}$ is not substantially changed. However, the theoretically predicted azimuthal velocity does not agree with the observed value, for the observed angle of about $1.5^{\circ}$ co-rotation between the direction of the solar wind and the radial direction gives about $10 \mathrm{~km}$ $\mathrm{sec}^{-1}$. From the directions of comet tails Brandt and Heise (1970) had found a similar value. An azimuthal velocity as high as $10 \mathrm{~km} \mathrm{sec}^{-1}$ at the orbit of the Earth would give a considerably higher transport of angular momentum. The exerted torque would be of the order of $7 \times 10^{30}$ dyne $\mathrm{cm}^{-1}$, assuming $V_{\varphi} \propto \sin \theta$. Such a large torque would decelerate the rotation of the entire Sun in $10^{10} \mathrm{yr}$ and the outer part of the convection zone in $10^{9} \mathrm{yr}$.

There still remains the difficulty of understanding how the solar wind can attain this high azimuthal velocity. Weber and Davis (1967b) as well as Meyer and Pfirsch (1969) have re-examined the problem with inclusion of viscosity and an anisotropic pressure tensor. Taking into account the latter, Meyer and Pfirsch obtain for the azimuthal velocity at large distances from the Sun:

$$
V_{\varphi}=\frac{\Omega r_{A}^{2}}{r}\left[1-\frac{r^{2}}{r_{A}^{2}} \frac{B_{r}^{2}}{4 \pi \varrho V_{r}^{2}}\left(1-\frac{p_{\|}-p_{\perp}}{B^{2} / 4 \pi}\right)\right]
$$

where $p_{\|}$and $p_{\perp}$ are the pressure components parallel and perpendicular to the magnetic field respectively.

This shows that an anisotropy of the pressure can increase the tendency towards co-rotation beyond $5 R_{\odot}$ and can give a much higher azimuthal velocity near the orbit of the Earth. For instance if the solar wind is near the limit of the 'firehose' instability with

$$
p_{\|}-p_{\perp} \approx B^{2} / 4 \pi
$$


then the azimuthal velocity would be of the order of

$$
V_{\varphi} \approx r \Omega r_{A}^{2} / r^{2}=7.5 \mathrm{~km} \mathrm{sec}^{-1}
$$

Schubert and Coleman (1968) have examined the transport of angular momentum from the Sun by hydromagnetic waves and showed that this mechanism may also be important in producing co-rotation of the plasma. Finally, it should be mentioned that the interaction of fast and slow streams can give a higher rotational velocity directed towards co-rotation, as Siscoe et al. (1968) have shown.

The problem of transport of angular momentum from a star to the surrounding interstellar gas had been investigated some time ago by Lüst and Schlüter (1955). They did not take into account the corpuscular radiation from the star, but assumed that force-free (or nearly force-free) fields connected to the star would be strong enough to enforce co-rotation out to some distance from the star. The angular momentum would be transported to distances from which turbulent friction would be effective.

Schatzman (1959) later connected this with the existence of a stellar wind, and he pointed out that if gas emitted by a star is magnetically constrained to co-rotation with the star out to distances large compared to the stellar radius, then a small amount of mass-loss would yield a significant loss of angular momentum, because of the effective increase of the moment of inertia of the gas during the outflow.

Mestel (1968) has linked this general picture with the theory of a quasi-stationary stellar wind, introducing important modifications to include the presence of strong centrifugal and magnetic fields. Mestel assumes a poloidal stellar field, which near the star should be roughly dipolar and strong enough to force the flow to follow the field and to keep the gas approximately co-rotating with the star. Farther out the gas flow drags the field, and each element approximately conserves its angular momentum. The two regions will be separated by a limiting field-line, the one where at the equator the wind speed just equals the local Alfvén speed. The theory is not applicable to the Sun and similar stars since the lines of force in the interplanetary space are not part of the Sun's polar field but emerge from near-equatorial regions with somewhat stronger fields than the polar regions. But Mestel's theory should describe the phenomena for a star contracting towards the main sequence, either down the Hayashi track, with the bulk of the star convective, or on the subsequent KelvinHelmholtz-type path, with the outer layers still strongly convective, and so still generating a corona.

Mestel showed that such a star can lose a substantial part of its angular momentum without losing a large percentage of its mass if the stellar magnetic field is just strong enough to keep the gas beneath the coronal base co-rotating with the star.

\section{Fluctuations, Discontinuities and Waves}

The observations of the solar wind and particularly of the imbedded magnetic fields show that there exist fluctuations in the plasma and magnetic fields which reflect the 'turbulent' structure of the motion. Furthermore one can distinguish different forms 
of discontinuities - sometimes shock waves have been identified and sometimes other waves. Very probably these phenomena are important in understanding the nature of the solar wind, in particular for the energy transport and the energy distribution with respect to the direction of the magnetic field as well as among the different constituents of the solar wind.

In analyzing the observed data one has to take into account that the Alfvén velocity in the wind at $1 \mathrm{AU}$ is typically $60 \mathrm{~km} \mathrm{sec}^{-1}\left(5 \times 10^{-5} \mathrm{G}\right.$ and $4 \mathrm{H}$-atom $\left.\mathrm{cm}^{-3}\right)$ and the thermal velocity is 15 to $100 \mathrm{~km} \mathrm{sec}^{-1}\left(10^{4}\right.$ to $\left.5 \times 10^{5} \mathrm{~K}\right)$. Both these velocities are small compared to the 300 to $700 \mathrm{~km} \mathrm{sec}^{-1}$ observed for the velocity of the wind. Thus the observed temporal variations can be transformed directly into spatial variations along the solar wind direction. The frequency $f$ of a fluctuation corresponds to the wavelength $\lambda=V / f$ in the radial direction where $V$ is the wind velocity.

Finally it should be mentioned that most observations of fluctuations refer to magnetic fields since the time resolution of the magnetometers is at present much higher than that of particle detectors. However, for a real understanding of the observed phenomena measurements of the other quantities are also needed.

The power spectra of the three magnetic field components and of the total field show about the same dependence on $f$, and normally the lowest power is in the magnitude of the field, indicating that the fluctuations are transverse waves. The power in the radial component is usually less, by a factor of almost two, than the power in the two transverse components. Therefore the fluctuations below about $1 \mathrm{~Hz}$ could well be transverse hydromagnetic waves. Waves with a frequency of $10^{-1} \mathrm{~Hz}$ have a wavelength of the order of $500 \mathrm{~km}$ (assuming a propagation velocity of $50 \mathrm{~km} \mathrm{sec}^{-1}$ ) and appear as fluctuations with a frequency $f \geqslant 1 \mathrm{~Hz}$ in the fixed frame of reference.

Below $10^{-5} \mathrm{~Hz}$ the power spectrum is flat. The spectrum at these very low frequencies originates mainly in time variations on the Sun and in features which co-rotate with the Sun. At higher frequencies the power spectrum declines with increasing $f$. There seems to be a change in the power spectrum over the years; the results so far are: 1962: $p \propto f^{-1}$ for $10^{-5} \leqslant f \leqslant 10^{-2} ; 1965: p \propto f^{-3 / 2}$ for $10^{-4}<f<10^{0}$ and, 1966: $p \propto f^{-1}$ to $p \propto f^{-3 / 2}$ for $10^{-6}<f \leqslant 10^{-4}$ and $p \propto f^{-2}$ for $10^{-4}<f<10^{-1}$. The spectrum is the same on active, intermediate, and quiet days. On active days the r.m.s. fluctuations are in excess of the large-scale field, and even in quiet days the fluctuations have occasional peaks comparable to $B$.

Scintillation of radio point sources is due to fluctuations in the interplanetary electron density. Observations indicate that the fluctuations have a correlation length of about 100 to $200 \mathrm{~km}$ corresponding to frequencies of about 1 to $10^{-1} \mathrm{~Hz}$. However, fluctuations in the magnetic field have a correlation length of about $2 \times 10^{6} \mathrm{~km}$ according to a recent investigation by Jokipii and Hollweg (1970). This length is defined as the characteristic scale beyond which the two-point correlation function falls rapidly towards zero. It corresponds to the point below which the frequency spectrum is nearly flat. Jokipii and Hollweg show that the large value of the correlation length is nevertheless in agreement with the observed interplanetary scintillation and that the 100 to $200 \mathrm{~km}$ scale refers to the 'inner scale' of the fluctuations of that wavelength 
below which there is little power. Most probably the observed fluctuations supply significant energy to the solar wind. In Section 3 I mentioned that the hydromagnetic waves are strongly damped. Therefore it is unlikely that waves with wavelengths less than about $2 \times 10^{6} \mathrm{~km}\left(f \geqslant 2 \times 10^{-4} \mathrm{~Hz}\right)$ will reach the Earth. Thus the longer wavelengths (lower frequencies) may be of solar origin but the rest of the spectrum, apparently, must originate in space. The mechanism proposed by Jokipii and Davis (see Section 3) may be responsible for the occurrence of these fluctuations.

The other mechanism which may explain the fluctuations at higher frequencies $\left(f \geqslant 10^{-3} \mathrm{~Hz}\right)$, is based on thermal anisotropies in the wind. These have been observed, but it is not yet clear whether they are able to drive the instabilities, since the theoretical interpretation of the observation is rather complicated. In summary only the following should be stated here. If the temperature parallel to the magnetic field $T_{\|}$exceeds the temperature perpendicular to the magnetic field $T_{\perp}$, we may expect the so-called 'firehose' instability. If one takes into account the resonance effect at the ion cyclotron frequency and the effect of the finite ion cyclotron radius, the instability can occur only when the total pressure of the gas exceeds the magnetic pressure. Under normal conditions $\left(n=5 \mathrm{~cm}^{-3}\right.$ and $\left.T_{p} \approx 5 \times 10^{4} \mathrm{~K}\right)$ the ion pressure is too small, and the ion anisotropy cannot drive the firehose instability, except during very active times when the temperature is very high. But the electrons are very hot and they may be able to produce both instabilities, namely the resonance instability occurring near the ion cyclotron frequency, and the 'firehose' instability. In this way the observation of fluctuations with high frequencies $\left(f>10^{-3} \mathrm{~Hz}\right)$ might be understood.

Besides these general fluctuations, individual fluctuations have been observed such as shock waves, shear planes, and oscillatory waves. But we shall not discuss them here.

\section{The Transition into the Interstellar Space}

\section{A. THE TRANSITION SHOCK FRONT}

The solar wind will finally mix with the interstellar gas. The related transition has been studied by several investigators (Axford et al., 1963; Parker, 1961; Hundhausen, 1968a; Brandt, 1964). Davis (1955) pointed out that the dynamical pressure of the solar wind, which falls off as $r^{-2}$, will be balanced at some heliocentric distance $r_{s}$ by the galactic magnetic field. Since the solar wind has supersonic velocities, it has to pass through a collisionless shock transition at $r_{s}$ (Parker, 1962, 1966). The dynamical pressure of the solar wind is about

$$
p_{s} \approx 2 \times 10^{-8} \frac{1}{r^{2}} \text { dyne } \mathrm{cm}^{-2}
$$

whereas the interstellar pressure is of the order $p_{i} \approx 10^{-12}$ dyne $\mathrm{cm}^{-2}$ (this is the sum of magnetic pressure, gas pressure, and cosmic-ray pressure). These values of $p_{i}$ and $p_{s}$ give $r_{s}=100 \mathrm{AU}$. The main uncertainty in this estimate is due to our imperfect knowledge of $p_{i}$ in the neighborhood of the Sun. But other effects - to be discussed below - enter too. At $100 \mathrm{AU}$ the solar wind density before the shock front would be 
about $10^{-4}$ proton $\mathrm{cm}^{-3}$. The shock raises the temperature to about $10^{7} \mathrm{~K}$ by thermalization of kinetic energy of mass motion; the density increases by a factor 4 if the ratio of specific heats is $\frac{5}{3}$. If other processes take place in this transition, the density can be higher.

\section{B. THE SOLAR HII REGION}

It certainly would make a difference whether the solar wind would be embedded in a $\mathrm{HI}_{\mathrm{I}}$ or in a HII region. The Sun is a rather cool star so that its Strömgren sphere will have a radius much less than a parsec. Williams (1965) has calculated the size of the solar Hil region taking into account the observed far-ultraviolet solar spectrum and assuming that the interstellar gas is at rest with respect to the Sun. It turns out that the outer boundary of the solar Hil region is not very sharp, but drops off gradually, a consequence of the presence of penetrating radiation with wavelengths around $200 \AA$. The ionization falls to $50 \%$ at $1500 \mathrm{AU}$ if $n_{i}=1$ atom $\mathrm{cm}^{-3}\left(n_{i}=\right.$ density of the interstellar gas), at $400 \mathrm{AU}$ if $n_{i}=10$ atom $\mathrm{cm}^{-3}$ and at $120 \mathrm{AU}$ if $n_{i}=100$ atom $\mathrm{cm}^{-3}$. According to this calculation the shock transition would lie inside the Hil region. The solar wind passing through the shock front would remain ionized due to the large recombination times.

\section{THE EFFECT OF CHARGE EXCHANGE WITH NEUTRAL INTERSTELLAR ATOMS}

The surrounding interstellar gas is moving with respect to the Sun with a velocity of $20 \mathrm{~km} \mathrm{sec}^{-1}$ towards the solar apex near $\alpha=18^{h}$ and $\delta=+30^{\circ}$. In addition it may fall towards the Sun due to the solar gravitation. At $10^{3}$ AU the gravitational escape velocity is only of the order of $1 \mathrm{~km} \mathrm{sec}^{-1}$ where the radiation pressure in the solar Ly- $\alpha$ line has been taken into account. If we assume that the gas is neutral at large distances from the Sun $\left(r>10^{4} \mathrm{AU}\right)$ the neutral hydrogen atoms will still come quite close to the Sun before they are ionized either by photoionization or by charge exchange with the solar wind. The photoionization time scale $\tau_{p h}$ is about

$$
\tau_{p h} \approx r^{2} / \alpha \quad[\mathrm{sec}]
$$

where $r=$ distance from the Sun in AU and $\alpha=$ photoionization rate at $1 \mathrm{AU}=10^{20}$ $\mathrm{cm}^{2} \mathrm{sec}^{-1}$. The neutral atoms will be ionized by photoionization between 5 to $10 \mathrm{AU}$ from the Sun depending on the direction with respect to the apex. The time scale for charge exchange $\tau_{c h}$ is given by the lifetime of a neutral atom in a plasma of $N$ atom $\mathrm{cm}^{-3}$

$$
\tau_{c h} \approx 10^{7} / N \quad[\mathrm{sec}]
$$

for velocities of $10^{7}$ to $10^{8} \mathrm{~cm} \mathrm{sec}^{-1}$. With numbers chosen by Parker one obtains a radius $r_{c h}$ of about $30 \mathrm{AU}$ which an inward-moving neutral hydrogen atom will reach before it is ionized by charge exchange with the solar wind.

We see that charge exchange can influence the solar wind quite appreciably and the wind might be degraded [i.e., neutralized, Ed.] before it reaches the shock front. This is possible when the flux of neutral interstellar atoms is as large or larger than the flux 
of ions in the solar wind. Thus serious degradation cannot occur unless there is at least one interstellar atom supplied for each wind ion. The wind flux $V N_{0}$ falls to the level of the interstellar flux of neutral atoms $N_{\mathrm{H}} U$ at

$$
r_{d}=r_{0}\left(\frac{V N_{0}}{U N_{\mathrm{H}}}\right)^{1 / 2}
$$

With $V=400 \mathrm{~km}, N_{0}=5$ atom $\mathrm{cm}^{-3}, U=3 \mathrm{~km} \mathrm{sec}^{-1}(400 \mathrm{~K})$ and $N_{\mathrm{H}}=1 \mathrm{~cm}^{-3}$ we get $r_{d}=15 \mathrm{AU}$. The values of $r_{d}$ and $r_{c h}$ suggest that the solar wind could be seriously degraded by interstellar hydrogen at $30 \mathrm{AU}$. This effect can bring the shock front closer to the Sun than is estimated above. Therefore, taking into account all the uncertainties mentioned, we conclude that the shock front may be found anywhere between 30 and $300 \mathrm{AU}$.

The charge exchange will convert solar wind protons into fast neutral hydrogen atoms and interstellar hydrogen atoms into relatively slow ions. If this occurs mainly outside the shock front, the fast or 'hot' neutral hydrogen atoms will have almost random direction. Ultimately the ions and electrons will disappear by radiative recombination but this is a very slow process and it requires a large volume. The lifetime for radiative recombination $\tau_{r}$ for a hydrogen plasma is given by

$$
\tau_{r} \approx 3 \times 10^{21}\left(T^{3 / 4} / n^{2}\right) \quad[\mathrm{sec}]
$$

If the recombination is in equilibrium with the production one obtains for $T=10^{4} \mathrm{~K}$ and $n=1 \mathrm{~cm}^{-3}$ a radius of the order of $10^{3}$ AU.

In the region between this outer boundary and the shock front, the lines of force of the solar magnetic field will mix with those of the interstellar field. Plasma-instabilities, such as the Rayleigh-Taylor instability, could be of importance.

With respect to stars other than the Sun I should finally mention the discussion by Newman and Axford (1968) of an isothermal corona expanding to such great distances that recombination occurs. This study may be of interest for stars with high surface temperature, such as planetary nebulae, with an outflow of $50 \mathrm{~km} \mathrm{sec}^{-1}$ and $10^{4}$ atom $\mathrm{cm}^{-3}$. In such a case a recombination front occurs before one passes through a shock transition.

\section{References}

Axford. W. J.: 1968, Space Sci. Rev. 8, 331.

Axford, W. J., Dessler, A., and Gottlieb, B.: 1963, Astrophys. J. 137, 1268.

Barnes, A.: 1968, Astrophys. J. 154, 751.

Barnes, A.: 1969, Astrophys. J. 155, 311.

Brandt, J. C.: 1964, Icarus 3, 253.

Brandt, J. C. and Heise, J.: 1970, Astrophys. J. 159, 1057.

Clauser, F. H.: 1960, Contribution from the Johns Hopkins University AFOSR TN 60-1386.

Davis, L.: 1955, Phys. Rev. 100, 1440.

Dessler, A. J.: 1967, Rev. Geophys. 5, 1.

Deutsch, A.: 1958, Astron. J. 63, 49.

Deutsch, A.: 1960, in Stars and Stellar Systems, Vol. VI (ed. by J. Greenstein), University of Chicago

Press, Chicago, p. 543. 
Deutsch, A.: 1961, in Aerodynamic Phenomena in Stellar Atmospheres (ed. by R. N. Thomas), Zanichelli, Bologna, p. 238.

Deutsch, A.: 1969, in Mass Loss from Stars (ed. by M. Hack), Reidel, Dordrecht, The Netherlands, p. 1.

Deutsch, A.: 1969, in Mass Loss from Stars (ed. by M. Hack), Reidel, Dordrecht, The Netherlands, p. 1.

Egidi, A., Pizella, G., and Signorini, C.: 1969, J. Geophys. Res. 74, 2807.

Hartle, R. E. and Sturrock, P.A.: 1968, Astrophys. J. 151, 1155.

Hundhausen, A. J.: 1968a, Planetary Space Sci. 16, 783.

Hundhausen, A. J.: 1968b, Space Sci. Rev. 8, 690.

Hundhausen, A. J., Gilbert, H. E., and Bame, S. J.: 1968, Astrophys. J. Lett. 152, L3.

Jokipii, J. R. and Davis, L.: 1969, Astrophys. J. 156, 1101.

Jokipii, J. R. and Hollweg, H. V.: 1970, Astrophys. J. 160, 745.

Lüst, R.: 1962, Space Sci. Rev. 1, 522.

Lüst, R. and Schlüter, A.: 1955, Z. Astrophys. 38, 190.

Mestel, L.: 1968, Monthly Notices Roy. Astron. Soc. 138, 359.

Meyer, P., Parker, E. N., and Simpson, J. A.: 1956, Phys. Rev. 104, 768.

Meyer, F. and Pfirsch, D.: 1969, Kleinheubacher Berichte 243.

Mustel', E.: 1964, Space Sci. Rev. 3, 319.

Ness, N. F.: 1968, Ann. Rev. Astron. Astrophys. 6, 79.

Newman, R. C. and Axford, W. J.: 1968, Astrophys. J. 151, 1145.

O'Gallagher, J. J. and Simpson, J. A.: 1967, Astrophys. J. 147, 819.

Ogilvie, K. W. and Wilkenson, T. D.: 1969, Solar Phys. 8, 435.

Parker, E. N.: 1958, Astrophys. J. 128, 664.

Parker, E. N.: 1961, Astrophys. J. 134, 20.

Parker, E. N.: 1962, Planetary Space Sci. 9, 461.

Parker, E. N.: 1963, Interplanetary Dynamical Processes, Interscience, New York.

Parker, E. N.: 1965, Space Sci. Rev. 4, 666.

Parker, E. N.: 1966, Planetary Space Sci. 14, 371.

Parker, E. N.: 1969, Space Sci. Rev. 9, 325.

Schatzman, E.: 1959, IAU Symposium No. 10, Cambridge University Press, Cambridge, p. 129.

Schubert, G. and Coleman, P. J.: 1968, Astrophys. J. 153, 943.

Simpson, A. J. and Wang, J.: 1967, Astrophys. J. Lett. 149, L73.

Siscoe, G. L., Davis, L., Coleman, P. J., Smith, E. J., and Jones, D. E.: 1968, J. Geophys. Res. 73, 61. Sturrock, P. A. and Hartle, R. E.: 1966, Phys. Rev. Lett. 16, 628.

Weber, E. J. and Davis, L.: 1967a, Astrophys. J. 148, 217.

Weber, E. J. and Davis, L.: 1967b, Trans. Am. Geophys. Union 48, 171.

Weymann, R.: 1960, Astrophys. J. 132, 380.

Weymann, R.: 1963, Ann. Rev. Astron. Astrophys. 1, 97.

Wilcox, J. M.: 1968, Space Sci. Rev. 8, 258.

Williams, R. E.: 1965, Astrophys. J. 142, 314. 